\title{
Experimental investigation of the ECRH stray radiation during the start-up phase in Wendelstein 7-X
}

\author{
Dmitry Moseev ${ }^{1, a}$, Heinrich Laqua ${ }^{1}$, Stefan Marsen ${ }^{1}$, Torsten Stange ${ }^{1}$, Harald Braune ${ }^{1}$, Volker Erckmann ${ }^{1}$, Florian \\ Gellert $^{1,2}$, Johann Wilhelm Oosterbeek ${ }^{3}$, Uwe Wenzel ${ }^{1}$, and the W7-X Team \\ ${ }^{1}$ Max-Planck-Institut für Plasmaphysik, Greifswald, Germany \\ ${ }^{2}$ Ernst-Moritz-Arndt-Universität Greifswald, Greifswald, Germany \\ ${ }^{3}$ Eindhoven University of Technology, Eindhoven, The Netherlands
}

\begin{abstract}
Electron cyclotron resonance heating (ECRH) is the main heating mechanism in the Wendelstein 7-X stellarator (W7-X). W7-X is equipped with five absolutely calibrated sniffer probes that are installed in each of the five modules of the device. The sniffer probes monitor energy flux of unabsorbed ECRH radiation in the device and interlocks are fed with the sniffer probe signals. The stray radiation level in the device changes significantly during the start-up phase: plasma is a strong microwave absorber and during its formation the stray radiation level in sniffer probes reduces by more than $95 \%$.

In this paper, we discuss the influence of neutral gas pressure and gyrotron power on plasma breakdown processes.
\end{abstract}

\section{Introduction}

Wendelstein 7-X (W7-X) is an optimized stellarator with $n=5$ symmetry operating in Greifswald, Germany. Electron cyclotron resonance heating $(\mathrm{ECRH})$ is the main heating mechanism in the device [1-3]. In its first experimental campaign, OP1.1, it was equipped with $4 \mathrm{MW}$ of ECRH power as a sole source of heating. Plasma was started also by means of ECRH.

In the highly reflective vacuum vessel of W7-X, unabsorbed microwave radiation can reach high energy fluxes (up to $350 \mathrm{~kW} / \mathrm{m}^{2} / \mathrm{MW}$ [4]) when there is no plasma. Therefore, studying the start-up processes is of high importance for the safety of the machine and its diagnostics. Both helium and hydrogen were used as fueling gasses for the experiments. The first plasma was produced in helium on 10.12.2015 and the fueling gas was changed to hydrogen on 03.02.2016. Plasma breakdown times for the two gasses are discussed in this contribution.

In Section 2 plasma breakdown is defined; the influence of neutral gas density and power on the delay time and the density evolution are shown in Section 3; Section 4 concludes the paper.

\section{Definition of plasma start-up time}

The plasma start-up time is usually defined as time from the beginning of heating, needed for the $H_{\alpha}$ emission to reach its maximum. It was shown [5] that the maximum of the $H_{\alpha}$ emission corresponds to the moment when interferometer starts detecting first plasma in the vessel and

\footnotetext{
a e-mail: dmitry.moseev@ipp.mpg.de
}

shows that the line integrated density is greater than zero. This is often referred to as a delay time. An alternative approach is to define the plasma start-up time by the moment when its capable of efficiently absorbing microwaves. We identify this moment by the drop of sniffer signal in module 3 by $90 \%$ from its maximum value. The module three is used in the analysis because of its location: it is the most remote from the ECRH launchers located in modules 1 and 5. Therefore, it is not influenced by the directly reflected ECRH beam. The two different definitions are illustrated in Fig. 1. There the time traces from 02.02.2016, 15:39 UTC show (from top to bottom) the total RF power injected into the vessel, stray radiation energy flux in module 3 , and line integrated density measured by interferometer. The zero moment on time axis denotes the start of ECRH operation. The left vertical bar shows the breakdown time defined by the plasma density and the right vertical bar shows the breakdown time defined by good microwave absorption.

In this contribution, we work with both of these definitions.

\section{Influence of the input power and neutral gas pressure on delay time and plasma evolution}

In Fig. 2 the plasma breakdown time, defined by interferometer is plotted against neutral gas pressure in module 5 , measured by a manometer $[\mu A]$. The ion current is directly proportional to the neutral gas pressure. The upper panel depicts the results for helium plasmas and 


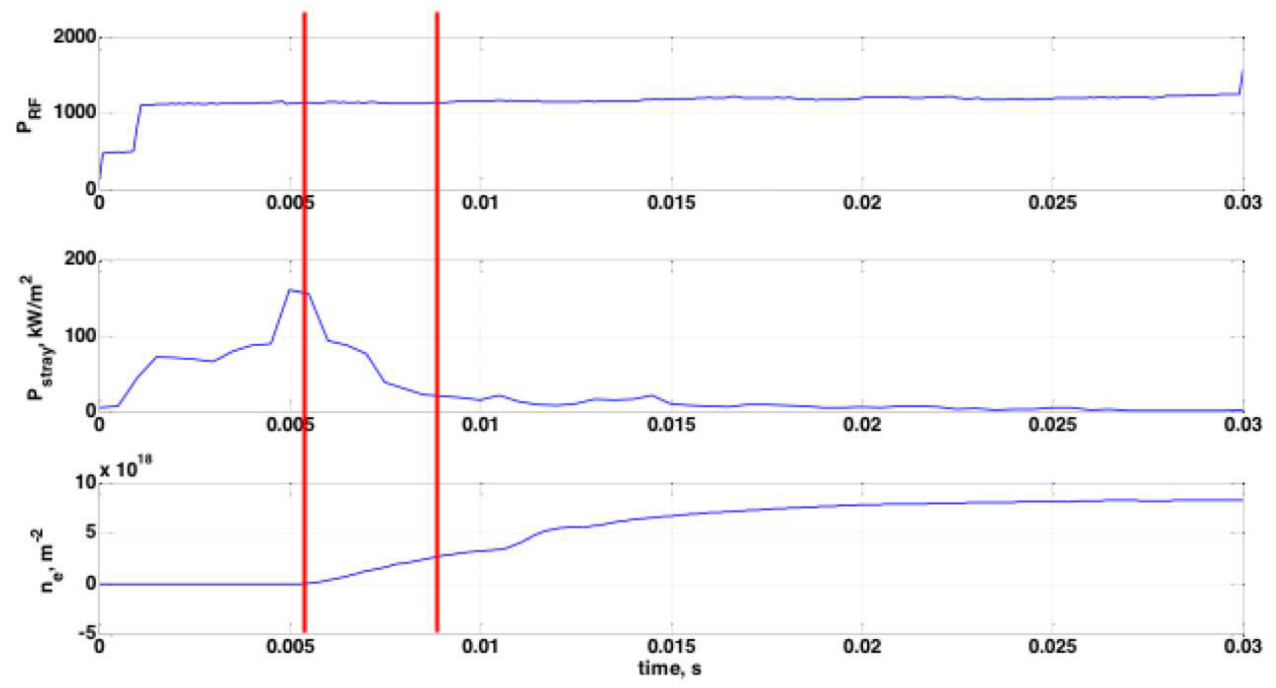

Figure 1. Definition of plasma breakdown. The example takes time traces from the experiment conducted on 02.02.2016 at 15:39 UTC. The upper panel shows total RF power injected by the ECRH system $[k W]$; the middle panel shows the energy flux of stray microwave radiation in module $3\left[\mathrm{~kW} / \mathrm{m}^{2}\right]$; the lower panel shows line integrated density measured by interferometer $\left[\mathrm{m}^{-2}\right]$. Zero time corresponds to the start of ECRH heating. The most left vertical bar indicates the plasma breakdown time defined by interferometer, when first plasma appears in the vessel. The most right vertical bar defines the start-up time by good microwave absorption, when the recorded stray radiation energy flux drops by $90 \%$ from its maximum value.

the lower panel shows the start-up times for hydrogen plasmas. Different power levels of ECRH are symboland color-coded. Counter-intuitively, the start-up times for helium plasmas are on average much longer than the corresponding start-up time for hydrogen. This is most likely due to poor conditioning of W7-X in the beginning of the OP1.1 campaign. A lot of impurities, particularly hydrogen and oxygen, were diagnosed spectroscopically in the beginning of the operational phase, and cleaning by means of the glow discharge was unavailable. The typical time traces of five experiments in helium (red) and five experiments in hydrogen plasmas are displayed in Fig. 3. One can clearly see, that the hydrogen plasma starts earlier and the state of good microwave absorption is achieved earlier as well.

Another interesting observation is that no dependence of plasma breakdown time on neutral density can be identified.

However, when the plasma start-up time is defined by good microwave absorption, the negative trend in the breakdown time versus neutral gas pressure in the launcher module is clearly visible. The corresponding graph for hydrogen plasmas is shown in Fig. 4. This behavior shows no impact of input ECRH power on the breakdown time which might indicate that the power dependence is saturated.

The dependence of density evolution on the neutral gas pressure in the launching module is positive and shown in Fig. 5. In this figure, the derivative of line integrated density is defined as $n_{e}^{\text {good.abs }} /\left(\tau_{\text {good.abs }}-\tau_{\text {breakdown }}\right)$. In accordance with the results for the start-up time, we do not observe the dependence of the density derivative over time as a function of power, as it is shown in Fig. 6.

Previously it was shown, that the upper estimate for the start-up time in W7-X should not exceed several tens milliseconds [6]. The results from the OP1.1 experimental campaign show that the plasma breakdown needs less than the estimated upper limit.

\section{Conclusions}

In total more than 800 experiments were conducted in the first experimental campaign OP1.1 at W7-X. Albeit poor conditioning, the ECRH plasma start-up proved to be very reliable. The delay time was always shorter than $30 \mathrm{~ms}$ and typically less than $15 \mathrm{~ms}$ for hydrogen plasma. We did not observe the dependence of the delay time on neutral gas pressure. However, the breakdown time defined by good microwave absorption is inversely proportional to the neutral gas pressure. It should be noted that the dependence is not very strong. No significant impact of the ECRH power is seen on the start-up process. The line integrated density evolves faster when the neutral gas pressure is higher, and yet again no effect of the ECRH is visible. These all might indicate that the power effect is saturated at lower values.

\section{Acknowledgments}

This work has been carried out within the framework of the EUROfusion Consortium and has received funding from the Euratom research and training programme 2014-2018 under grant agreement No 633053. The views and opinions expressed herein do not necessarily reflect those of the European Commission. 

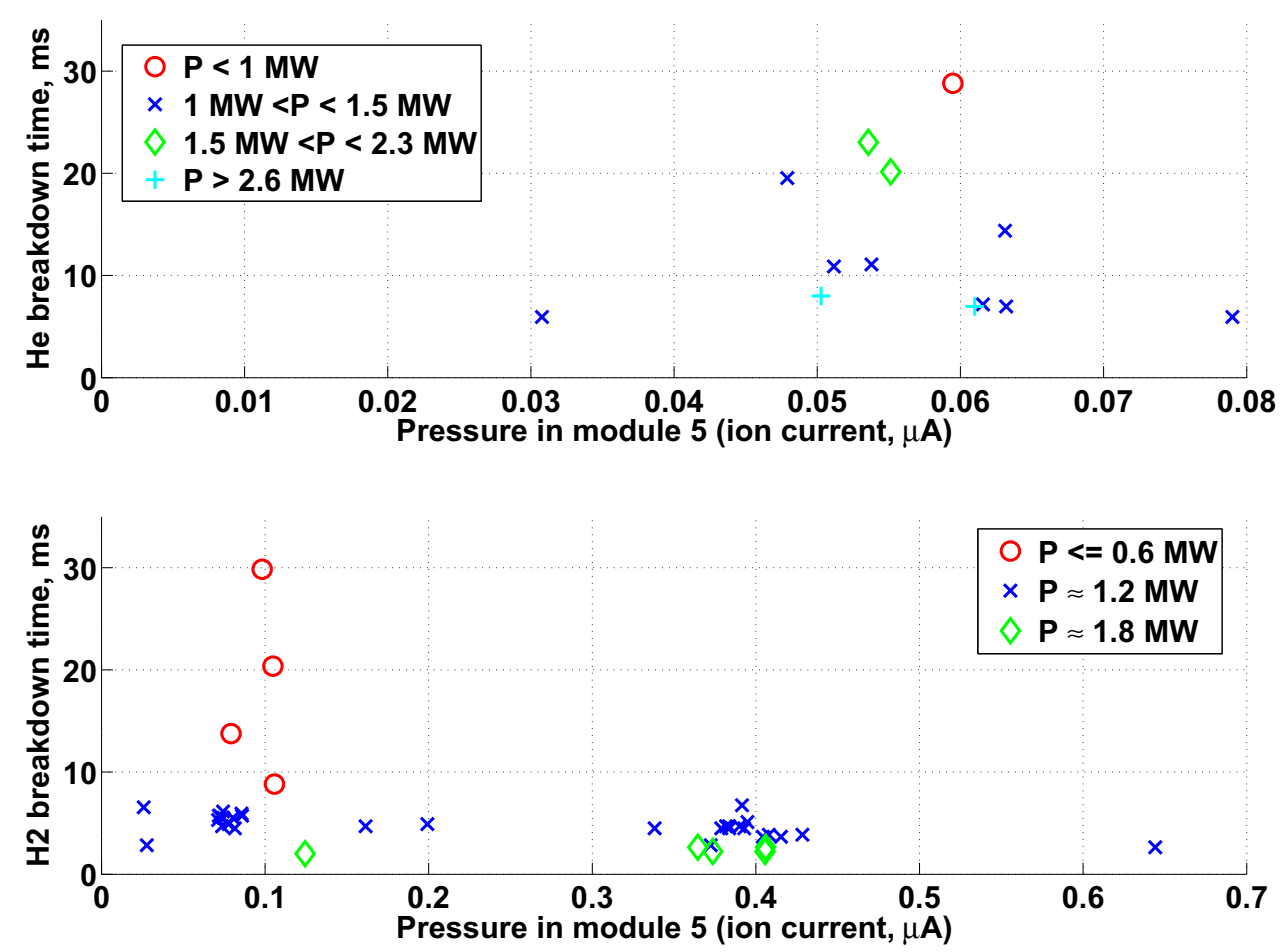

Figure 2. Plasma breakdown time, defined by the interferometer, is plotted against the manometer readings in module 5 of W7-X, where the ECRH launcher is located. The upper panel shows the start-up time for helium and the lower panel depicts start-up time for hydrogen plasmas. Power levels are symbol- and color-coded.
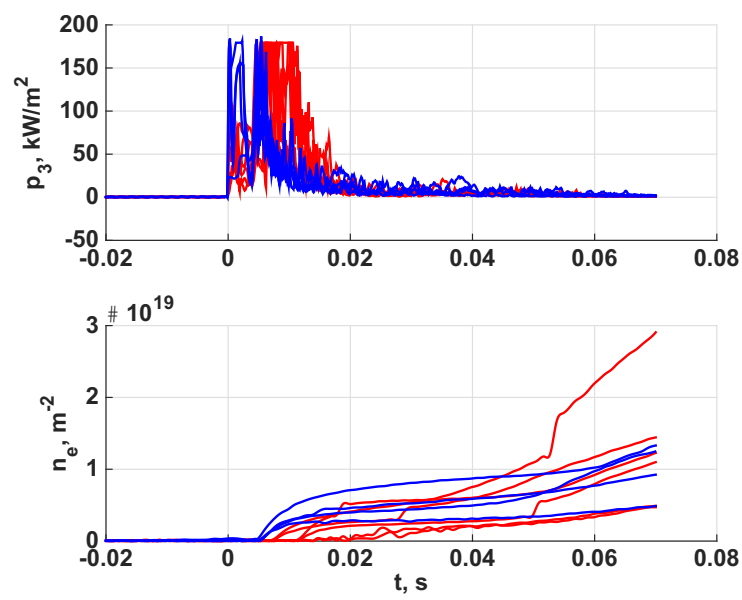

Figure 3. Time traces showing typical experiments in hydrogen plasmas (blue) and helium plasmas (red). Upper panel depicts temporal evolution of stray radiation energy flux in module 3; the lower panel shows temporally resolved line integrated density. Zero on time axis corresponds to the start of ECRH.

\section{References}

[1] V. Erckmann, P. Brand, H. Braune, G. Dammertz, G. Gantenbein, W. Kasparek, H.P. Laqua, H. Maass-

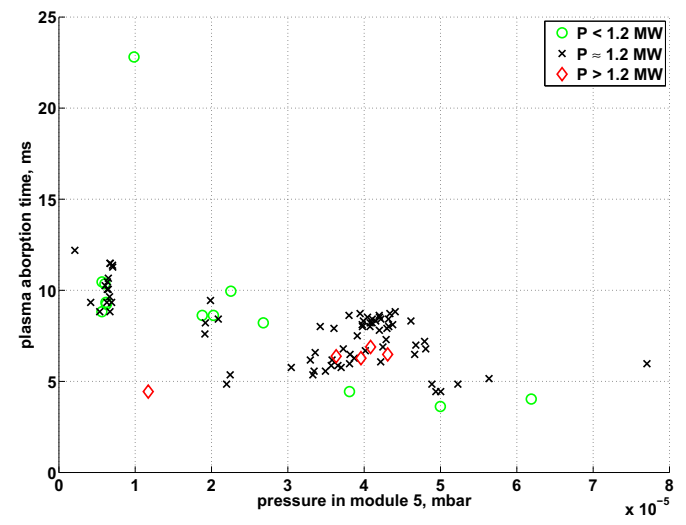

Figure 4. Plasma breakdown time in hydrogen plasmas, defined by good microwave absorption, is plotted against the manometer readings in module 5 of $\mathrm{W} 7-\mathrm{X}$, where the ECRH launcher is located. Power levels are symbol- and color-coded.

berg, N.B. Marushchenko, G. Michel et al., Fusion Science and Technology 52, 291 (2007)

[2] V. Erckmann, P. Brand, H. Braune, G. Dammertz, G. Gantenbein, W. Kasparek, H.P. Laqua, G. Michel, M. Thumm, M. Weissgerber, The $140 \mathrm{GHz}, 10 \mathrm{MW}$, CW ECRH Plant for W7-X: A Training Field for ITER, in Fusion Energy 2006 (International Atomic Energy 
Agency, Chengdu, 2007), pp. IT/2-4Rd

[3] J. Geiger, R.C. Wolf, C. Beidler, A. Cardella, E. Chlechowitz, V. Erckmann, G. Gantenbein, D. Hathiramani, M. Hirsch, W. Kasparek et al., Plasma Physics and Controlled Fusion 55, 014006 (2013)

[4] D. Moseev, H.P. Laqua, S. Marsen, T. Stange, H. Braune, V. Erckmann, F. Gellert, J.W. Oosterbeek,

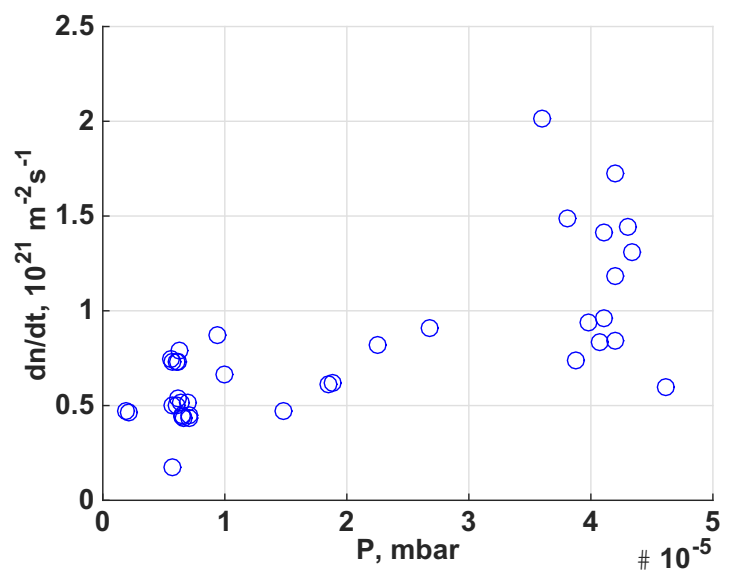

Figure 5. Derivative of the line integrated density in the period between plasma breakdown and the moment of good absorption for hydrogen plasmas plotted as a function of the average neutral gas in the launching module.

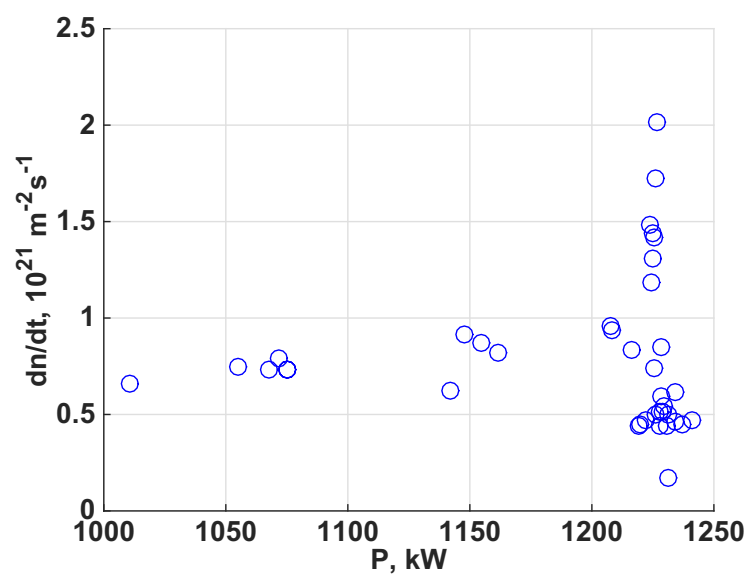

Figure 6. Derivative of the line integrated density in the period between plasma breakdown and the moment of good absorption for hydrogen plasmas plotted as a function of the ECRH power injected into the vessel.
Review of Scientific Instruments 87, 083505 (2016)

[5] A. Cappa, F. Castejón, F. Tabarés, D. Tafalla, Nuclear Fusion 41, 363 (2001)

[6] M. Preynas, D. Aßmus, H. Igami, S. Kado, S. Kobayashi, S. Kubo, H. Laqua, T. Mutoh, K. Nagasaki, M. Otte et al., EPJ Web of Conferences 87, 02005 (2015) 